\title{
Retrospective analysis of clinical results and predictors of response in chemo-naïve patients with advanced gastric cancer treated with S-1, an oral fluoropyrimidine derivative, as single-agent chemotherapy
}

\author{
Kan Yonemori, Yasuhiro Shimada, Ayumu Goto, Takashi Ura, Tatsuhiro Arai, Tetsuya Hamaguchi, \\ Kei Muro, Yasuhide Yamada, and Kuniaki Shirao \\ Department of Medical Oncology, National Cancer Center Hospital, 5-1-1 Tsukiji, Chuo-ku, Tokyo 104-0045, Japan
}

\begin{abstract}
Background. Despite the fact that there are only a few reports of phase II studies, S-1 is widely used in single-agent or combination therapies for patients with advanced gastric cancer in Japan. We retrospectively analyzed the effectiveness of S-1 as single-agent chemotherapy for patients with advanced gastric cancer.

Methods. A total of 119 patients with advanced or recurrent gastric cancer were treated with $\mathrm{S}-1$ as first-line monochemotherapy from September 1999 to March 2003 at the National Cancer Center Hospital. S-1 was administered orally twice daily, at a standard dose of $80 \mathrm{mg} / \mathrm{m}^{2}$ per day for 28 days, followed by a 14-day rest.

Results. One hundred and eleven patients were analyzed retrospectively. The overall response rate was $26.1 \%(29 / 111$; $\mathbf{9 5} \%$ confidence interval [CI], $17.8 \%$ to $34.1 \%$ ). Median time to progression and median overall survival were 141 days (95\% CI, 108 to 175 days) and 378 days (95\% CI, 310 to 447 days), respectively. The response rate of ascites, according to the Japanese classification of gastric carcinoma, was $36.8 \%$ (14/38; 95\% CI, 25.4\% to 56.6\%). Among all of the pretreatment variables examined, hemoglobin level and the presence of lymph node metastasis were related to the response.

Conclusion. Single-agent chemotherapy of S-1 for chemonaïve patients with advanced gastric cancer was modestly effective and well-tolerated in the outpatient setting.
\end{abstract}

Key words S-1 - Chemotherapy · Advanced gastric cancer · Response $\cdot$ Predictive factor

\section{Introduction}

S-1 is an oral antitumor agent that combines three pharmacological agents: tegafur, which is a prodrug of 5fluorouracil (5-FU), 5-chloro-2-4,-dihydroxypyridine

Offprint requests to: $\mathrm{K}$. Yonemori

Received: May 11, 2004 / Accepted: July 26, 2004
(CDHP), which inhibits dihydropyrimidine dehydrogenase (DPD) activity, and potassium oxonate (Oxo), which reduces gastrointestinal toxicity. Two late phase II studies of S-1 in advanced gastric cancer conducted in Japan showed overall response rates of $44 \%$ and $49 \%$, times to progression of 135 and 158 days, and overall survival times of 207 and 250 days, respectively [1,2]. There were no cases of grade 4 hematological toxicities or nonhematological toxicity, and $5 \%$ or fewer patients were affected by grade 3 toxicities.

At present, S-1 is widely used in single-agent or combination therapies in the setting of palliative, adjuvant, or neo-adjuvant chemotherapy of gastric cancer [3]. S-1 has a larger area under the curve for peritoneal dissemination and ascites than for plasma [4], and thus might be effective for prolonging the survival of gastric cancer patients with peritoneal dissemination [5-7].

We evaluated the effectiveness of S-1 for chemonaive patients with advanced gastric cancer in the outpatient setting and analyzed the pretreatment factors related to the response.

\section{Patients and methods}

A total of 119 patients with advanced or recurrent gastric cancer were treated with S-1 alone, as first-line chemotherapy from September 1999 to March 2003 at the National Cancer Center Hospital. From among these patients, we selected patients as subjects for the present study if they fulfilled the following eligibility criteria: (1) a diagnosis of histologically or cytologically proven gastric cancer; (2) no previous chemotherapy or radiotherapy; (3) adequate bone marrow and organ functions (leukocytes, $\geqq 3000 / \mu \mathrm{l}$; neutrophils, $\geqq 1500 / \mu \mathrm{l}$; hemoglobin, $\geqq 8.0 \mathrm{~g} / \mathrm{dl}$; platelets, $\geqq 100000 / \mu \mathrm{l}$, total bilrubin, $<2.0 \mathrm{mg} / \mathrm{dl}$; aspartate aminotransferase [AST], $<100$ IU/l; alanine aminotransferase [ALT], <100 IU/l; serum creatinine, $\leqq 1.5 \mathrm{mg} / \mathrm{dl}$ ); and (4) all patients were 
required to provide written informed consent for treatment.

S-1 was administered orally at a standard dose of $80 \mathrm{mg} / \mathrm{m}^{2}$ per day by administering the following dosages, twice daily, after breakfast and dinner: body surface area less than $1.25 \mathrm{~m}^{2}, 40 \mathrm{mg} ; 1.25$ to $1.5 \mathrm{~m}^{2}, 50 \mathrm{mg}$; more than $1.5 \mathrm{~m}^{2}, 60 \mathrm{mg}$. One cycle of therapy consisted of S-1 treatment twice daily for 28 days, followed by a 2 -week off-treatment period. The dosage was reduced in patients with evidence of hematological toxicity of grade 3 or greater or nonhematological toxicity of grade 2 or greater. This cycle was repeated unless disease progression, patient refusal, or unacceptable toxicity occurred.

The responses to treatment of metastatic lesions were assessed according to World Health Organization (WHO) criteria [8]. Metastatic lesions were evaluated by radiographic examination. Primary lesions and bone metastases were not considered as measurable sites. Patients without measurable lesions were classified as "not evaluable (NE)".

The response of ascites was evaluated by abdominal computed tomography (CT), based on the following specific criteria according to the Japanese classification of gastric carcinoma [9]: (1) disappearance of ascites: disappearance of ascites visualized by CT scan for at least 4 weeks; (2) decrease of ascites: apparent decrease of ascites visualized by CT scan for at least 4 weeks; and (3) no response of ascites: no change of ascites volume visualized by CT scan [9]. Toxicity was evaluated according to the National Cancer Institute common toxicity criteria (NCI-CTC) version 2.0. Time to progression (TTP) was measured from the first day of treatment until disease progression or the last day of the follow-up period without disease progression, and overall survival (OS) time was measured from the first day of treatment until death or the last day of the follow-up period. Median time to progression and median overall survival were estimated using the Kaplan-Meier method. Data were analyzed using SPSS 11.0J (SPSS, Tokyo, Japan).

\section{Factors analyzed}

Eleven pretreatment variables were investigated for their relationship to tumor response. The pretreatment variables examined were chosen by considering possible effects on the clinical course, as indicated by previous investigations [10-13], as well as those suggested from our own experience. The relationship of response to the following 11 categorized variables was studied: (1) Eastern Cooperative Oncology Group (ECOG) performance status (PS; 0 or 1); (2) histology (differentiated type or undifferentiated type); (3) macroscopic classification [9] (infiltrating type [type 4] or other types); (4) site of disease (classified as follows: primary lesion [present or absent]); (5) lymph node metastasis (present or absent); (6) liver metastasis (present or absent); (7) peritoneal disease, which included peritoneal dissemination and ascites (present or absent); (8) hemoglobin level (hemoglobin; male, $\geqq 13.7 \mathrm{~g} / \mathrm{dl}$ or $<13.7 \mathrm{~g} / \mathrm{dl}$; female, $\geqq 11.3 \mathrm{~g} / \mathrm{dl}$ or $<11.3 \mathrm{~g} / \mathrm{dl}$ ); (9) serum C-reactive protein (CRP; $\geqq 1.0 \mathrm{mg} / \mathrm{dl}$ or $<1.0 \mathrm{mg} / \mathrm{dl}$ ); (10) serum lactate dehydrogenase (LDH; $\geqq 230 \mathrm{U} / 1$ or $<230 \mathrm{U} / 1$ ); and (11) serum carcinoembryonic antigen (CEA; $>5.0 \mathrm{ng} / \mathrm{ml}$ or $\leqq 5.0 \mathrm{ng} / \mathrm{ml})$.

\section{Statistical analysis}

We determined the pretreatment predictive factors for clinical response to S-1 therapy using the univariate $\chi^{2}$ test and multivariate logistic regression analysis. Variables with a univariate $P$ value of less than 0.1 were included in the multivariate analysis. A $P$ value of less than 0.05 was considered significant. Regression coefficients were estimated by the maximum likelihood method and model; addition was performed stepwise, using the likelihood ratio test for calculating the probability of a patient being a responder.

\section{Results}

One hundred and eleven of the 119 patients were enrolled in the present analysis. Eight patients were excluded from the study according to the eligibility criteria, on the basis of inadequate bone marrow and organ functions. Median follow-up time was 347 days, and follow-up time ranged from 42 to 1107 days. Patients' demographics are listed in Table 1. The median age was 61 years (range, 30 to 84 years). All patients had an ECOG performance status of 0 or 1 . Most patients $(67.6 \%)$ showed the undifferentiated type of histology. Eighteen patients (16.2\%) had infiltrating-type macroscopic classification. Ninety-four patients were evaluable for the response according to WHO criteria. Fifty-nine patients had primary gastric tumor and 52 patients had peritoneal disseminaton with or without ascites. Thirty-eight patients were evaluated for the response of ascites according to the Japanese classification of gastric carcinoma [9].

The median number of courses of treatment was 3 (total number of courses, 546; range, 1 to 20). The response rates of all patients, and of evaluable patients, were $26.1 \%$ ( 2 complete response [CR] and 27 partial response [PR] in 111 patients); $95 \%$ confidence interval (CI), $17.8 \%$ to $34.1 \%$ ) and $30.8 \%$ (2 CR and 27 PR in 94 patients), respectively. The median time to progression (TTP) was 141 days (Fig. 1; 95\% CI, 108 to 175 days). The median overall survival (OS) was 378 days (95\% 
Table 1. Patient demographics

\begin{tabular}{lc} 
& No. and percentage (\%) of patients \\
\hline Number & 711 \\
Male/Female & $79 / 32$ \\
Median age, years (range) & \\
ECOG performance status & $48(43.2 \%)$ \\
0 & $63(56.8 \%)$ \\
1 & \\
Histology & $36(32.4 \%)$ \\
Differentiated type & $75(67.6 \%)$ \\
Undifferentiated type & $18(16.2 \%)$ \\
Infiltrating type of macroscopic classification & \\
Site of disease & $59(53.2 \%)$ \\
Primary lesion & $68(61.3 \%)$ \\
Lymph nodes & $52(46.8 \%)$ \\
Peritoneal disease & $37(33.3 \%)$ \\
Liver metastasis & $7(6.3 \%)$ \\
Lung metastasis & $4(3.6 \%)$ \\
Bone metastasis & $9(8.1 \%)$ \\
Other & $10(9 \%)$ \\
Number of involved sites & $29(26.1 \%)$ \\
4 & $35(31.5 \%)$ \\
3 & $37(33.3 \%)$ \\
2 &
\end{tabular}

ECOG, Eastern Cooperative Oncology Group

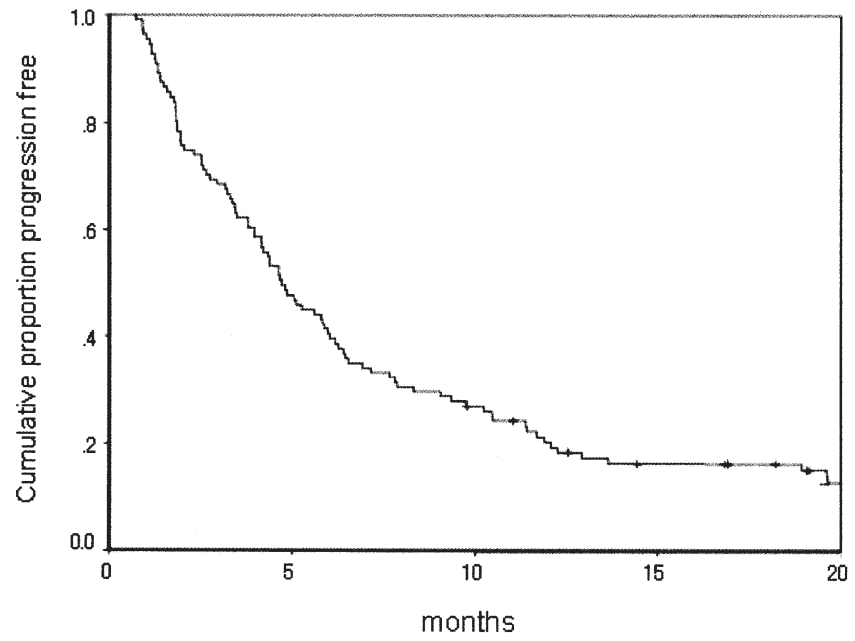

Fig. 1. Kaplan-Meier analysis of time to progression $(n=111)$

CI, 310 to 447 days), with a 2-year survival rate of $20 \%$ (Fig. 2). The response rate of ascites was 36.8\% (14/38; $95 \%$ CI, $25.4 \%$ to $56.6 \%$ ).

The toxicity profiles are listed in Table 2. Major adverse reactions included myelosuppression and gastrointestinal toxicities, and the incidence of grade 3 adverse reactions was $21.6 \%$ (24/111). No grade 4 toxicities observed. Twelve patients required dose reduction due to toxicities. There were no treatmentrelated deaths.

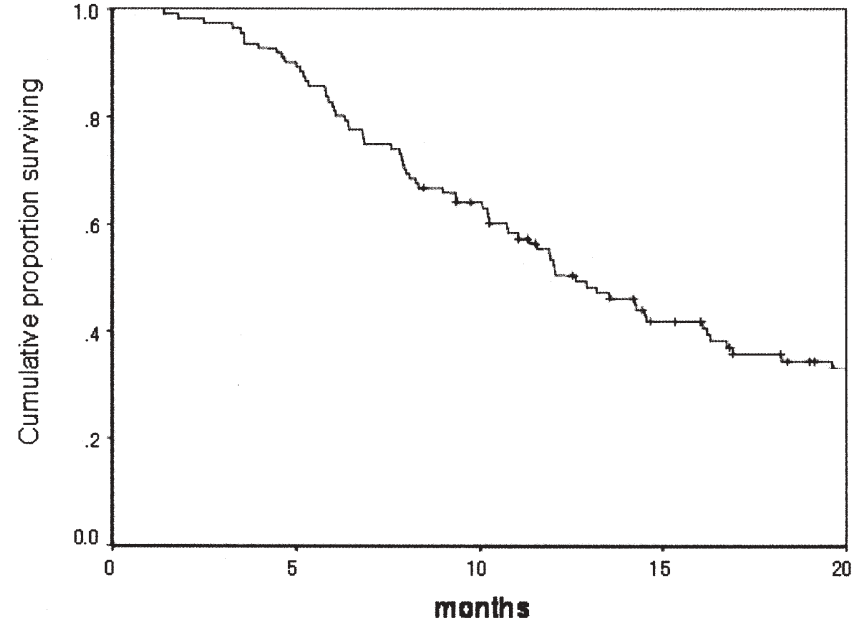

Fig. 2. Kaplan-Meier analysis of overall survival $(n=111)$

After disease progression, 60 patients (54.0\%) had second-line chemotherapy, as follows: irinotecan (CPT11)-containing combination chemotherapy $(26 \% ; 29 /$ 111), taxane single-agent chemotherapy $(21 \% ; 23 / 111)$, and 5-fluorouracil (5-FU)-containing chemotherapy (7.2\%; 8/111). Third-line, fourth-line, and fifth-line chemotherapy regimens were administered in 23,7 , and 3 patients, respectively.

In the univariate analysis, the hemoglobin level was significantly associated with tumor responsiveness to 
Table 2. Toxicity per patient (546 courses in 111 patients)

\begin{tabular}{|c|c|c|c|c|}
\hline & \multicolumn{4}{|c|}{$\begin{array}{l}\text { Maximum grade (NCI-CTC ver 2.0); } \\
\text { percentage of patients }\end{array}$} \\
\hline & 1 & 2 & 3 & 4 \\
\hline Leukocytes & 27 & 13 & 1 & 0 \\
\hline Neutrophils & 20 & 15 & 7 & 0 \\
\hline Hemoglobin & 49 & 32 & 2 & 0 \\
\hline Platelets & 2 & 0 & 0 & 0 \\
\hline Bilirubin & 39 & 8 & 3 & 0 \\
\hline SGOT & 41 & 5 & 1 & 0 \\
\hline SGPT & 35 & 8 & 1 & 0 \\
\hline Creatinine & 4 & 0 & 1 & 0 \\
\hline Appetite loss & 47 & 15 & 5 & 0 \\
\hline Nausea & 40 & 5 & 1 & - \\
\hline Diarrhea & 32 & 9 & 5 & 0 \\
\hline Fatigue & 61 & 8 & 0 & 0 \\
\hline Stomatitis & 32 & 2 & 1 & - \\
\hline Hand-foot syndrome & 25 & 2 & 1 & - \\
\hline Pigmentation & 45 & 2 & - & - \\
\hline Rash & 25 & 4 & 1 & - \\
\hline Fever & 7 & 1 & - & - \\
\hline Maximum toxicity per patient ${ }^{\mathrm{a}}$ & 27 & 51 & 22 & 0 \\
\hline
\end{tabular}

${ }^{a}$ Frequency of maximum grade of toxicity per patient

S-1 therapy (Table 3). Pretreatment variables were subsequently analyzed using the multivariate logistic regression model, and we found that normal hemoglobin level and the presence of lymph node metastasis were independently related to the response $(P=0.006$ and $P$ $=0.026$, respectively).

\section{Discussion}

In advanced gastric cancer, systemic chemotherapy using 5-FU increases the median survival to 7-10 months, from 3-4 months with the best supportive care [3]. A randomized controlled trial recently conducted in Japan revealed that the response rate, TTP, and OS with 5FU alone were $11 \%, 1.9$ months, and 7.1 months, respectively, and that these values were not significantly different from those obtained with 5-FU-based combination chemotherapy [14]. Therefore, 5-FU alone was considered the reference chemotherapy in this disease for the current investigation in Japan.

We retrospectively analyzed 111 consecutive patients with advanced gastric cancer treated with S-1 as firstline chemotherapy in the clinical setting. In this analysis, the response rate was $26 \%$, which was lower than that previously reported. We think that this may have been due to selection bias, because the two late phase II studies excluded patients without measurable lesions $[1,2]$. Also, those trials had strict eligibility requirements for bone marrow and organ functions because of the use of a novel agent for therapy. In the clinical setting, there were many patients who did not meet the eligibility criteria of the phase II trial. Therefore, selection bias may explain why the response rate in this study was lower than that in the two late phase II trials.

Several reports have demonstrated that S-1 was effective against peritoneal disease and undifferentiated type of histology, such as poorly differentiated, mucinous, and signet-ring cell carcinoma [2,4-7]. However, histological type did not have a significant influence on the responsiveness to S-1 therapy on either univariate or multivariate analysis in the present study. On the other hand, the median OS of 378 days in the present study was slightly longer than the OS values determined in the previous phase II studies. These differences may have been due to differences of population demographics and the salvage chemotherapy after S-1 failure.

In the phase II trials of S-1, Sakata et al. [1] reported that adverse reactions appeared in $78 \%$ of the patients, and the frequency of adverse reactions of grades 3 and 4 was $20 \%$, and Koizumi et al. [2] did not describe the frequency of toxicity per patient in detail. Thus, we think that the frequency of severe toxicity of grades 3 and 4 in this study was similar to that found in the late phase II trial [1]. The overall frequency of the maximum grade of toxicity per patient in the present study was higher than that in the late phase II trial. This may have been because the present study consisted of 546 courses in 111 patients, including 12 patients with potential 
Table 3. Univariate analysis of response to chemotherapy according to patient characteristics

\begin{tabular}{|c|c|c|c|}
\hline Variables & Number & Response rate (\%) & $P$ value \\
\hline \multicolumn{4}{|l|}{ Performance status } \\
\hline 1 & 63 & 28.6 & \multirow[t]{2}{*}{0.523} \\
\hline 0 & 48 & 22.9 & \\
\hline \multicolumn{4}{|l|}{ Histology } \\
\hline Undifferentiated type & 75 & 25.3 & \multirow[t]{2}{*}{0.820} \\
\hline Differentiated type & 36 & 27.8 & \\
\hline \multicolumn{4}{|l|}{ Macroscopic type } \\
\hline Infiltrating type & 18 & 16.7 & \multirow[t]{2}{*}{0.393} \\
\hline Other types & 93 & 28.0 & \\
\hline \multicolumn{4}{|l|}{ Site of disease } \\
\hline \multicolumn{4}{|l|}{ Primary lesion } \\
\hline Present & 59 & 32.1 & \multirow{2}{*}{0.195} \\
\hline Absent & 52 & 20 & \\
\hline \multicolumn{4}{|l|}{ Lymph nodes } \\
\hline Present & 68 & 32.4 & \multirow[t]{2}{*}{0.077} \\
\hline Absent & 43 & 16.3 & \\
\hline \multicolumn{4}{|l|}{ Liver metastasis } \\
\hline Present & 37 & 27.0 & \multirow[t]{2}{*}{1.00} \\
\hline Absent & 74 & 25.7 & \\
\hline \multicolumn{4}{|l|}{ Peritoneal disease } \\
\hline Present & 52 & 21.2 & \multirow[t]{2}{*}{0.287} \\
\hline Absent & 59 & 30.5 & \\
\hline \multicolumn{4}{|l|}{ Hemoglobin level ${ }^{\mathrm{a}}$} \\
\hline Normal & 33 & 42.4 & \multirow[t]{2}{*}{0.017} \\
\hline Low & 78 & 19.2 & \\
\hline \multicolumn{4}{|l|}{ CRP } \\
\hline$\geqq 1.0 \mathrm{mg} / \mathrm{dl}$ & 34 & 29.4 & \multirow[t]{2}{*}{0.643} \\
\hline$<1.0 \mathrm{mg} / \mathrm{dl}$ & 77 & 24.7 & \\
\hline \multicolumn{4}{|l|}{$\mathrm{LDH}$} \\
\hline$\geqq 230 \mathrm{U} / 1$ & 27 & 33.3 & \multirow[t]{2}{*}{0.327} \\
\hline$<230 \mathrm{U} / 1$ & 84 & 23.8 & \\
\hline \multicolumn{4}{|l|}{ CEA } \\
\hline$\geqq 5.0 \mathrm{ng} / \mathrm{ml}$ & 43 & 23.3 & \multirow[t]{2}{*}{0.661} \\
\hline$<5.0 \mathrm{ng} / \mathrm{ml}$ & 68 & 27.9 & \\
\hline
\end{tabular}

CRP, C-reactive protein; LDH, lactate dehydrogenase; CEA, carcinoembryonic antigen

${ }^{a}$ Normal, $\geqq 13.7 \mathrm{~g} / \mathrm{dl}$ in males and $\geqq 11.3 \mathrm{~g} / \mathrm{dl}$ in females; low, $<13.7 \mathrm{~g} / \mathrm{dl}$ in males and $<11.3 \mathrm{~g} / \mathrm{dl}$ in females

organ impairment (who would have been ineligible for late phase II trials) and 38 patients with ascites. In patients with ascites, third-space retention of an administered drug is associated with prolongation of the terminal drug half-life in plasma, presumably owing to the slow reentry of the sequestered drug into the bloodstream [15]. This effect may intensify the toxicities in patients with ascites, who frequently show more severe toxicities than patients without ascites $[16,17]$.

In the present study, the frequency of hand-foot syndrome was higher than that in previous studies [1,2]. The frequency of skin reaction (rash) was $8 \%$ to $16 \%$ in those previous studies, and it is difficult to distinguish rash from hand-foot syndrome, particularly that of grade 1 toxicity. Obvious hand-foot syndrome of grade 2 or greater occurred in only three patients in present study, and this frequency may be similar to that in those previous studies [1,2].
In any case, it was clear that S-1 single-agent therapy was well-tolerated in the outpatient setting and that the frequency of severe toxicity in S-1 therapy might be lower than that seen in intravenous infusion treatment for patients with advanced gastric cancer in clinical practice [10].

It was interesting that normal hemoglobin level and the presence of lymph node metastasis were predictive factors for the response to S- 1 on the multivariate analysis. As far as we know, no reports are currently available describing the hemoglobin level as a possible predictive factor for the clinical outcome in gastric cancer patients receiving chemotherapy $[10,14,18]$. Although the etiology is not well understood, anemia is a strong predictor of poorer survival in cancer patients with solid tumors or hematological malignancies $[13,19]$. In gastric cancer, anemia is frequently seen in patients with ulcerative primary lesions [20]. Moreover, S-1 was less effective in 
patients with low hemoglobin level in the present study. In the literature, normal hemoglobin level was reported to be an independent predictor of response to chemotherapy $[21,22]$. It is possible that hemoglobin level may be related to tumor burden [18]. In addition, low hemoglobin level is a major contributing factor to tumor hypoxia, which occurs when the tumor growth exceeds the ability of the local microvasculature to supply oxygen to the tumor cells [23]. Hypoxia renders solid tumors resistant to low-level radiation, chemotherapy, and photodynamic therapy [23].

Another positive predictor of response was the presence of lymph node metastasis, a category including all patients with lymph node metastasis. This categorized factor included 15 patients with lymph node metastasis as the only lesion. Thus, this factor included patients with low tumor burden compared to other categorized factors such as the presence of primary lesion, or liver metastasis. In the late phase II trials of S-1, the response rate in patients with metastatic lymph nodes was $44.8 \%$ to $57.0 \%$, depending on the organ. This was higher than the response rates of in patients with other disease sites $[1,2]$. These considerations may have influenced the analysis of predictors of response. However, it cannot be denied that the predictors of response may have been positive in our patient group by chance. Therefore, our findings need verification in an independent group of patients.

Although our study is not strictly comparable with previously reported studies, and provides insufficient evidence for S-1 as first-line chemotherapy, the relatively longer overall survival time and mild toxicity profiles observed in the present study lead us to consider that it may be important to examine combination therapy of S-1 and other drugs having different mechanisms of action and different efficacy spectra. To obtain clear evidence about first-line chemotherapy in advanced gastric cancer, we have joined the Japan Clinical Oncology Group (JCOG) 9912, three-arm randomized trial, which is comparing 5-FU alone, S-1 alone, and irinotecan plus cisplatin combination therapy, and we hope that the results of the JCOG 9912 will be conclusive.

In conclusion, single-agent chemotherapy of S-1 is effective and well-tolerated for chemo-naïve patients with advanced gastric cancer in the outpatient setting. Hemoglobin level and presence of lymph node metastasis were positive predictors of response.

\section{References}

1. Sakata Y, Ohtsu A, Horikoshi N, Sugimachi K, Mitachi Y, Taguchi T. Late phase II study of novel oral fluoropyrimidine anticancer drug S-1 in advanced gastric cancer patients. Eur J Cancer 1998;34:1715-20.
2. Koizumi W, Kurihara M, Nakano S, Hasegawa K. Phase II study of S-1, a novel oral derivative of 5-fluorouracil in advanced gastric cancer. Oncology 2000;58:191-7.

3. Cutsem EV, Haller D, Ohtsu D. The role of chemotherapy in the current treatment of gastric cancer. Gastric Cancer 2002;5(Suppl 1):17-22.

4. Mori T, Fujiwara Y, Yano M, Tamura S, Yasuda T, Takiguchi S, et al. Prevention of peritoneal metastasis of human gastric cancer cells in nude mice by S-1, a novel oral derivative of 5-fluorouracil. Oncology 2003;64:176-82.

5. Osugi H, Takada N, Takemura M, Kaseno S, Lee S, Ueno M, et al. Oral fluoropyrimidine anticancer drug TS-1 for gastric cancer patients with peritoneal dissemination. Oncol Rep 2002;9: 811-5.

6. Yoshida K, Nishimoto N, Kagawa Y, Hihara J, Inoue H, Kim R, et al. A new therapeutic approach to advanced and recurrent gastric cancer by TS-1. Gan to Kagaku Ryoho (Jpn J Cancer Chemother) 2001;28:1403-12.

7. Inaba Y, Watabe S, Ohe S, Kamio Y, Koyama M, Hayashi K, et al. The clinical effect of TS-1 in advanced and recurrent gastric cancer with peritoneal dissemination. Gan to Kagaku Ryoho (Jpn J Cancer Chemother) 2002;29:239-44.

8. World Health Organization: WHO handbook for reporting results of cancer treatment (offset publication no.48). Geneva: WHO; 1997.

9. Japanese Research Society for Gastric Cancer. Japanese classification of gastric carcinoma. Tokyo: Kanehara; 1999.

10. Yoshida M, Boku N, Ohtsu A, Muto M, Nagashima F, Yoshida S. Combination chemotherapy of irinotecan plus cisplatin for advanced gastric cancer: efficacy and feasibility in clinical practice. Gastric Cancer 2001;4:144-9.

11. Louvet C, Carrat F, Mal F, Mabro M, Beerblock K, Vaillant JC, et al. Prognostic factor analysis in advanced gastric cancer patients treated with hydroxyurea, leucovorin, 5-fluorouracil and cisplatin. Cancer Invest 2003;21:14-20.

12. Baba H, Okuyama T, Hiroyuki O, Anai H, Korenaga D, Maehara $\mathrm{Y}$, et al. Prognostic factors for noncurative gastric cancer: univariate and multivariate analyses. J Surg Oncol 1992;51:1048.

13. Caro JJ, Salas M, Ward A, Goss G. Anemia as an independent prognostic factor for survival in patients with cancer: a systemic, quantitative review. Cancer 2001;91:2214-21.

14. Ohtsu A, Shimada Y, Shirao K, Boku N, Hyodo I, Saito H, et al. Randomized phase III trial of fluorouracil alone versus fluorouracil plus cisplatin versus uracil and tegafur plus mitomycin in patients with unresectable gastric cancer: the Japan Clinical Oncology Group Study (JCOG9205). J Clin Oncol 2003;21:514.

15. Chabner Bam Stoller RG, Hande K, Jacobs S, Young RC. Methotrexate disposition in humans: case studies in ovarian cancer and following high dose infusion. Drug Metab Rev 1978;8:10717.

16. Aslam N, Marino CR. Malignant ascites. Arch Intern Med 2001; 161:2733-7.

17. Marincola FM, Schwartzentruber DJ. Malignant ascites. In: De Vita VT Jr, Hellman S, Rosenberg SA, editors. Cancer: principle and practice of oncology. 6th Ed. Philadelphia: Lippincott Wiliams and Wilkins; 2001. p. 2745-52.

18. Rougier P, Ducreux M, Mahjoubi M, Pignon JP, Bellefiqih S, Oliveira J, et al. Efficacy of combined 5-fluorouracil and cisplatinum in advanced gastric carcinomas. A phase II trial with prognostic factor analysis. Eur J Cancer 1994;30A:1263-9.

19. Van Belle SJ, Cocquyt V. Impact of haemoglobin levels on the outcome of cancers treated with chemotherapy. Crit Rev Oncol Hematol 2003;47:1-11.

20. Wanebo HJ, Kennedy BJ, Chmiel J, Steele G Jr, Winchester D, Osteen R. Cancer of the stomach. A patient care study by the American College of Surgeons. Ann Surg 1993;218:58392. 
21. Osterlind K, Hansen HH, Dombernowsky P, Hansen M, Anderson OK. Determinants of complete remission induction and maintenance in chemotherapy with or without irradiation of small cell lung cancer. Cancer Res 1987;47:2733-6.

22. Sengelov L, Kamby C, Geertsen P, Andersen LJ, Von der Maase H. Predictive factors of response to cisplatin-based chemotherapy and the relation of response to survival in patients with metastatic urothelial cancer. Cancer Chemother Pharmacol 2000;46:35764.

23. Vaupel P, Thews O, Hoeckel M. Treatment resistance of solid tumors: role of hypoxia and anemia. Med Oncol 2001;18:24359. 